\title{
TRANSITIVE STEINER AND KIRKMAN TRIPLE SYSTEMS OF ORDER 27
}

\author{
CHARLES J. COLBOURN, SPYROS S. MAGLIVERAS, AND RUDOLF A. MATHON
}

\begin{abstract}
There are 71 Steiner triple systems of order 27 whose automorphism groups are point-transitive, and there are 248 transitive Kirkman triple systems of order 27. Computational methods used to find these designs are outlined. The designs and some of their properties are presented.
\end{abstract}

\section{STEINER TRIPLE SYSTEMS AND THEIR GROUPS}

A Steiner triple system of order $v$, briefly $\operatorname{STS}(v)$, is a pair $(V, \mathscr{B})$ where $V$ is a set of $v$ elements and $\mathscr{B}$ is a set of 3-element subsets of $V$, with the property that every 2-subset of $V$ appears in exactly one subset of $\mathscr{B}$. Sets in $\mathscr{B}$ are triples. An automorphism of an $\operatorname{STS}(v)$ is a permutation on $V$ that maps each triple in $\mathscr{B}$ to a triple of $\mathscr{B}$, and the automorphism group is the group of all automorphisms of the STS. The STS is transitive if the automorphism group acts transitively on $V$; it is cyclic when the group contains $Z_{v}$ as a subgroup, and abelian if there is an abelian subgroup of the automorphism group that acts transitively on $V$.

Steiner triple systems with large automorphism groups, and in particular transitive STS, are studied in large part because they yield examples of Steiner triple systems with interesting "regularity." There is an extensive literature on cyclic STS [2], but for the closely related case of transitive STS, especially nonabelian STS, little is known.

Transitive STS of order 21 have been constructively enumerated [5]; there are seven cyclic designs and three other transitive ones. Subsequently, Tonchev [8] constructively enumerated the transitive $\operatorname{STS}(25)$; there are three over $Z_{5} \times Z_{5}$, in addition to the twelve over $Z_{25}$ known since the 1930s [1].

For order 27, there are five possible automorphism groups to be considered. Three are the abelian groups $Z_{3} \times Z_{3} \times Z_{3}, Z_{9} \times Z_{3}$, and $Z_{27}$. In addition, there are two nonabelian groups [3]. The first of these has all group elements of order 3, while the second has $Z_{9}$ as a subgroup. Every transitive STS(27) has (at least) one of these contained in its automorphism group. Cyclic STS(27)'s have been generated previously [1], as have the "1-rotational" STS(27)'s [7].

In this paper, we exhibit all transitive STS(27)'s. There are eight cyclic ones [1]; we find that there are 71 transitive STS(27) 's in total. In addition to presenting these designs, we give an extensive computational analysis of them.

Received June 21, 1990; revised January 15, 1991.

1991 Mathematics Subject Classification. Primary 05B07, 51E10. 
We list numbers of subdesigns, group orders, and whether or not the design is resolvable.

A parallel class in an STS is a spanning set of pairwise disjoint triples. A resolution is a partition of the triples of the STS into parallel classes. A Steiner triple system along with a resolution of it is a Kirkman triple system, or KTS. An automorphism of the STS is also an automorphism of a Kirkman triple system that it supports, provided the automorphism preserves the parallel classes of the resolution. In general, a Steiner triple system that is transitive can be resolvable in many ways; some Kirkman triple systems so arising may be transitive, while others are not. For a transitive STS, a transitive resolution (under a group $\Gamma$ ) is a resolution whose parallel classes are preserved under the action of $\Gamma$. A transitive KTS is a Kirkman triple system whose automorphism group acts transitively on elements (and, of course, preserves the resolution).

We examine all resolutions of the transitive STS $(27)$ that are preserved by one of the groups acting transitively on 27 elements; we establish that there are precisely 248 nonisomorphic transitive Kirkman triple systems of order 27 . We exhibit a compact representation of each, along with its group order.

Janko and van Trung [4] have previously found all 661 "2-rotational" KTS(27) 's, each necessarily having group order divisible by 13 . Only one of their designs is also transitive as a Kirkman triple system, and hence 247 of the designs we exhibit appear to be previously unpublished.

Before presenting the catalogues of designs, we outline the computational methods used. Let $\Gamma$ be one of the five groups of order 27 , presented as a transitive permutation group on $V$, a set of 27 elements. The action of $\Gamma$ partitions the 3-subsets of $V$ into orbits $T_{1}, \ldots, T_{t}$, and partitions the pairs into orbits $P_{1}, \ldots, P_{p}$. Now form a matrix $A=A_{23}(\Gamma)$, whose $(i, j)$ entry is the number of times a fixed pair in orbit $P_{i}$ appears in triples of orbit $T_{j}$. Let $U$ be a 0,1 -solution to the matrix equation $A U=1$. Then $U$ is the characteristic vector of a Steiner triple system whose automorphism group contains $\Gamma$.

Solutions of this matrix equation are in one-to-one correspondence with distinct STS(27)'s whose automorphism group contains $\Gamma$. Hence all such STS(27)'s can be found by solving a binary knapsack problem. However, we are interested only in nonisomorphic solutions. Applying a permutation $\pi$ in the normalizer of $\Gamma$ in $S_{2 y m}$ (the symmetric group on 27 symbols) carries an STS to an isomorphic, but possibly distinct, STS. Using the normalizer of $\Gamma$ to eliminate duplicates in the search for the solutions to the matrix equation substantially reduces the number of solutions to be examined. Finally, we find one representative of each isomorphism type, and its automorphism group, using the graph isomorphism program "nauty" of McKay [6].

Parallel classes are found as follows. Form a 117-vertex block nonintersection graph, having a vertex representing each block, and two vertices adjacent when the corresponding blocks are disjoint. A parallel class is a 9-clique in this graph. Each parallel class generates an orbit of parallel classes under the action of $\Gamma$. Such an orbit may have all parallel classes having no triples in common (a type1 class), or two parallel classes of the orbit may share a triple (a type-2 class). A transitive resolution contains only type-1 classes. To check resolvability (not necessarily transitive), form a graph whose vertices are the parallel classes, and make two vertices adjacent if the corresponding parallel classes have no common triple. A resolution is a 13-clique in this graph. In a typical case, the graph has 
thousands of vertices (parallel classes), and so we did not find all resolutions, but verified the existence or nonexistence of one.

Finding transitive resolutions is an easier matter. One first eliminates all type- 2 classes. Then the remaining parallel classes fall into orbits under $\Gamma$. We form a graph whose vertices are the orbits of parallel classes, where each vertex has a weight equal to the number of parallel classes in the orbit. Two vertices are adjacent if no parallel class in the orbit represented by one vertex shares a triple with a parallel class of the orbit represented by the second vertex. Then a transitive resolution (that is, a transitive Kirkman triple system) is a clique of weight 13 in this graph.

Finally, determining the nonisomorphic transitive Kirkman triple systems was again performed by nauty. The success of the approach here rests on the effective solution of two difficult problems: an integer knapsack problem, and a clique problem. These are the same problem in disguise; the approach used is a heuristic method that quickly prunes the number of cases to be considered.

To determine the solutions of the binary knapsack problem $A U=J$, we use an algorithm called SYNTH. At present, SYNTH is ideally suited for problems in which $A$ has several thousand columns but fewer than 100 rows, and determines all possible solutions. In this recursive algorithm, a column $X$ of $A$ which has a 1 in the first row is selected. All rows in which $X$ has a 1 are marked for deletion, and so are all columns of $A$ that are not orthogonal to $X$ (that is, those that have nonzero inner product with column $X$ ). A synthem is a pair of binary vectors indicating which rows are active (not marked for deletion), and which columns are active. Synthems are used to pass information about these "conceptual" differences recursively to the main function in SYNTH. The synthem passed is then used to prune the possible solution space. This pruning process conceptually yields submatrix $A^{\prime}$ of $A$ (without actually incurring the overhead of carrying out the deletions physically), and the algorithm recursively determines all solutions to subproblem $A^{\prime} U^{\prime}=J^{\prime}$. The above deletions are relative only to the subproblem indicated by the synthem. The algorithm proceeds to select the second column $X$ of $A$ with a 1 in $A$ 's first row, and the process continues until all 1's in row one of $A$ have been considered.

\section{TRANSITIVE STS OF ORDER 27}

In this section, we exhibit all 71 transitive Steiner triple systems of order 27. We report nonisomorphic solutions for each of the five groups; some designs are represented over more than one of the groups, but we assign each isomorphism type of design a unique number that is used throughout. When a design has a presentation over more than one group, we give a presentation of it over each relevant group.

For each design, the number of subdesigns has been determined; none has a subdesign of order 7 or 13, and the only admissible order for a subdesign is 9 . We report the number of subdesigns for each design under column "S."

It is an easy exercise to see that each design has a parallel class, since there is some orbit of nine triples in each solution. However, not all of the designs are resolvable. Under the column "Res," we report on resolvability of each design as follows. " $N$ " indicates that the design has no resolution at all. " $R$ " indicates that it is resolvable but has no transitive resolution under the action of the 
group. " $T$ " indicates that it has a transitive resolution, and the accompanying number is the number of such resolutions under the action of the group. In the case of design 50, the design has a unique resolution, and this resolution is transitive.

We have also computed the number of parallel classes in each design; this ranges from 478 (design 44) to 17641 (design 1). Design 1 is of course the affine geometry; hence, the fact that it maximizes the number of parallel classes, resolutions, transitive resolutions, and subdesigns, comes as no surprise.

Finally, we have also computed cycle structures [2] for each design. Design 1 has a 2-transitive automorphism group, and hence its cycle structure must be the same for all pairs of elements (i.e., it is uniform). Remarkably, design 2 is also uniform and has the same cycle structures as design 1 . None of the remaining 69 are uniform, although designs 3 and 6 have only two different cycle structures.

For compactness, we list the designs with element set $\{a, b, \ldots, y, z, A\}$, listing a block $\{a, b, c\}$ as $a b c$. We only list orbit representatives for each design. To obtain the full set of 117 blocks, one applies the group generated by the generators given in each case to find the orbits of the representative triples; their union is the block set of the design.

2.1. The nonabelian group of exponent three. We present here the results for the nonabelian group of order 27 having all group elements of order 3 . We use the set of three generators:

\author{
bcaxutmpjkirngfws Ayovehzqdl \\ drhealpicfmjxAzqgsbwtnukoyv \\ ftoljgazyqspbcndewumkixrvAh
}

There are forty nonisomorphic Steiner triple systems carried by this group; we present them here in compact form:

\begin{tabular}{|c|c|c|c|c|}
\hline \multicolumn{2}{|c|}{ No. Order } & $\mathbf{S}$ & Res & Orbit Representatives \\
\hline 1 & 303264 & 39 & T 729 & abc ade a f $g$ ahu aix ajp akA alq amo ant arv as $z$ awy \\
\hline 2 & 11664 & 12 & T 567 & abc ade a fg ahk aix ajp alq amo ant arv awy \\
\hline 3 & 486 & 3 & $\mathbf{R}$ & abc ade afh aix ajp alq amo ant arv as z awy \\
\hline 4 & 432 & 3 & T 15 & abc ade a f g ahi ak A alv amo ant awy \\
\hline 5 & 162 & 3 & T 27 & abc ade a f g ahi ak A alq amo ant arv as z awy \\
\hline 6 & 162 & 3 & $\mathbf{R}$ & abc ade a fh air a jp alq amo ant asz \\
\hline 7 & 81 & 3 & $\mathrm{~T} 21$ & abc ade af $g$ ahk aij alq amo arv awy \\
\hline 8 & 81 & 0 & T 6 & abc ade a f g ahi akm anw arv \\
\hline 9 & 81 & 0 & T 3 & abc ade af $g$ ahj ain aky asz \\
\hline 10 & 54 & 3 & $\mathbf{R}$ & abc ade afh aij alq amo arv as $z$ awy \\
\hline
\end{tabular}


$11543 \mathrm{R}$ abcade afhain ajpamoarvaszawy

12540 T 9 abcade afgahiaknalv amo

13543 T 5 abcade afgahk aijalrawy

$14543 \mathrm{R}$ abcadeafhain ajmarvasz

$15543 \mathrm{~T} 3$ abcadeafhaix ajvalnasz

$16543 \mathrm{R}$ abcadeafiahl ajsak Aawy

17270 T 5 abcade af gahiakm ant arv as z awy

18270 T 5 abcade afgahiakA alq amoans awy

$19270 \mathrm{R}$ abcadeafhaim ajpalq ant arvawy

$20270 \mathrm{~T} 3$ abcade af gahiakl amoans

21270 T 2 abcade afgahiakmans awy

22270 T 3 abcade afg ahiaknalq amz

$23270 \mathrm{~T} 1$ abcade afgahiaknalrasz

24270 T 2 abcadeafgahjaiqaknasz

$25270 \mathrm{~T} 1$ abcade afhaijalqamzawy

$26273 \mathrm{R}$ abcadeafhaijalraszawy

$27270 \mathrm{R}$ abcadeafhaijalvamoawy

$28270 \mathrm{~T} 1$ abcade afhaimajpaln arv

$29270 \mathrm{~T} 1$ abcade afhaim ajt alq awy

$30270 \mathrm{R}$ abcadeafhain ajpamzawy

$31270 \mathrm{R}$ abcadeafhaiqajwant arv

$32270 \mathrm{R}$ abcadeafhairajnalqamo

33270 T 2 abcade afh air ajp alt amo

$34270 \mathrm{~T} 1$ abcade afhaix ajmalt arv

$35270 \mathrm{~T} 1$ abcade afhaix ajmalvant

36270 T 2 abcadf ahm akl ans

37270 T 3 abc adf ahq aik amz

$38270 \mathrm{~T} 1$ abcadf aht aimakl

39270 T 2 abcadf ahv aim akn

40270 T 1 abcadf ahxakl amz

2.2. The nonabelian group of exponent nine. In this subsection, we treat the cases for the nonabelian group having a subgroup isomorphic to $Z_{9}$, having generators:

\author{
dule f ghijkamnopqrscAvwxyztb \\ bcaxAwtvzuygkf jeidhlosnrmqp
}

This group carries 18 nonisomorphic triple systems, as follows: 


\begin{tabular}{|c|c|c|c|c|}
\hline \multicolumn{2}{|c|}{ No. Order } & $\mathbf{S}$ & Res & Orbit Representatives \\
\hline 1 & 303264 & 39 & Т 39 & abcady aeu afiagvanz aqw \\
\hline 2 & 11664 & 12 & T9 & abnad A aex afiagy \\
\hline 3 & 486 & 3 & $\mathbf{R}$ & $a b f$ ad $A$ aex agy aqw \\
\hline 41 & 81 & 0 & T 5 & abladwaeuafiagn \\
\hline 42 & 27 & 0 & $\mathbf{R}$ & abl adf ags am $A$ anz \\
\hline 43 & 27 & 0 & $\mathrm{~T} 2$ & abl adg aex amA anz \\
\hline 44 & 27 & 0 & $\mathrm{~T} 1$ & abl adi aex amz $a q w$ \\
\hline 45 & 27 & 0 & $\mathbf{R}$ & abl adiaex amA anz \\
\hline 46 & 27 & 0 & $\mathbf{R}$ & abladjags amz aqw \\
\hline 47 & 27 & 0 & $\mathrm{~T} 1$ & abladjags am $A$ anz \\
\hline 48 & 27 & 0 & $\mathrm{~N}$ & ablads aeh amw anz \\
\hline 49 & 27 & 0 & $\mathrm{~T} 1$ & abladsaei amw anz \\
\hline 50 & 27 & 0 & $\mathrm{~T} 1$ & abladvaeh anz $a q A$ \\
\hline 51 & 27 & 0 & $\mathrm{~T} 2$ & abladvaeianzaqA \\
\hline 52 & 27 & 0 & T 3 & abladvaezafiagw \\
\hline 53 & 27 & 0 & T 5 & abladwaezafiagm \\
\hline 54 & 27 & 0 & $\mathrm{~T} 4$ & abladx aeo afiagr \\
\hline 55 & 27 & 0 & $\mathrm{~T} 4$ & abladx aeuafiagz \\
\hline
\end{tabular}

2.3. The group $Z_{3} \times Z_{3} \times Z_{3}$. For the elementary abelian group $Z_{3}^{3}$, we use the three generators:

bcaefdhigkljnomqrptuswxv z Ay
defghiabcmnopqrjklvwxyzAstu
jklmnopqrstuvwxyzAabcdefghi

This group carries five nonisomorphic triple systems, all of which are carried as well by at least one of the groups already handled.

\author{
No. Order $S$ Res Orbit Representatives \\ 130326439 T 81 abcadhajtamAapx \\ 21166412 T99 abcadhajvakxalw \\ $\begin{array}{lllll}3 & 486 & 3 & \text { T } 81 \text { abcadgaeiafjakwal Aaoz }\end{array}$ \\ $\begin{array}{lllll}6 & 162 & 3 & \text { T } 81 \text { abcadhajvakxalz }\end{array}$ \\ $\begin{array}{lllll}41 & 81 & 0 & \text { T } 29 \text { abcadgaejafmalvaozaqx }\end{array}$
}


2.4. The group $Z_{9} \times Z_{3}$. Here we give solutions for the abelian group $Z_{9} \times Z_{3}$, with generators:

$$
\begin{aligned}
& \text { dlmef ghijkanuopqrstbvwxyzAc } \\
& \text { bcalnopqrstmduvwxyz Ae fghijk }
\end{aligned}
$$

This group carries 15 nonisomorphic triple systems, as follows:

$$
\begin{aligned}
& \text { No. Order S Res Orbit Representatives } \\
& 130326439 \text { T } 27 \text { abcadqael afiagnaoyarv } \\
& 2 \quad 1166412 \text { T9 advadtaepafiagq } \\
& 3486 \quad 3 \quad \mathbf{R} \text { abfadAaewagxaoy } \\
& \begin{array}{lllll}
5 & 162 & 3 & \text { T9 abcadqaelafiaguaoyarv }
\end{array} \\
& \begin{array}{lllll}
7 & 81 & 3 & \text { T } 3 & \text { abvadqaelafiagu }
\end{array} \\
& \begin{array}{lllll}
8 & 81 & 0 & \text { T } 3 & \text { abmadraepafiagy }
\end{array} \\
& 981 \quad 0 \quad \text { T3 abmadraezafiagy } \\
& \begin{array}{lllll}
56 & 54 & 3 & \mathbf{R} & \text { abf adtaepag } A \text { aoy }
\end{array} \\
& \begin{array}{lllll}
57 & 54 & 3 & \mathrm{R} & \text { abfadtaezagAaoy }
\end{array} \\
& \begin{array}{lllll}
58 & 27 & 0 & \mathrm{~T} 2 & \text { abmadgaesanxarv }
\end{array} \\
& \begin{array}{lllll}
59 & 27 & 0 & \text { T } 1 & \text { abmadiaepaoyaqy }
\end{array} \\
& \begin{array}{lllll}
60 & 27 & 0 & \text { T } 2 & \text { abmadiaesanxarv }
\end{array} \\
& \begin{array}{lllll}
61 & 27 & 0 & \mathrm{~T} 1 & \text { abmadiaesanyarv }
\end{array} \\
& \begin{array}{lllll}
62 & 27 & 0 & \text { T } 5 & \text { abmadraepafiags }
\end{array} \\
& \begin{array}{lllll}
63 & 27 & 0 & \text { T } 5 & \text { abmadraezafiags }
\end{array}
\end{aligned}
$$

2.5. The group $Z_{27}$. The cyclic designs have been known for over fifty years [1]. We include them here for completeness, using the generator:

$$
\text { bcde f ghijklmnopqrstuvwxyzAa }
$$

There are eight nonisomorphic cyclic STS(27):

No. Order S Res Orbit Representatives

$$
\begin{array}{lllll}
64 & 27 & 0 \mathrm{~N} & \text { abdaelafpagoajs } \\
65 & 27 & 0 \mathrm{~N} & \text { abdaelafpagtajs } \\
66 & 27 & 0 \mathrm{R} & \text { abdaelafragoajs } \\
67 & 27 & 0 \mathrm{~T} 2 & \text { abdaelafragtajs } \\
68 & 27 & 0 \mathrm{~T} 2 & \text { abdaeoaflahpajs } \\
69 & 27 & 0 \mathrm{~N} & \text { abdaeoaflahtajs } \\
70 & 27 & 0 \mathrm{~N} & \text { abdaerafvahpajs } \\
71 & 27 & 0 \mathrm{R} & \text { abdaerafvahtajs }
\end{array}
$$

\section{TRANSITIVE KiRKMAN TRIPLE SYSTEMS}

Forty-nine of the Steiner triple systems exhibited in $\S 2$ can be resolved in such a way that the Kirkman triple system is also transitive. In the appendix 
(Supplement section at the end of this issue), we exhibit all nonisomorphic transitive Kirkman triple systems of order 27. There are 248 altogether. Hence we resort to a compact representation of the systems.

We list only those parallel classes that suffice to generate the thirteen parallel classes under the action of the groups (as given in \$2). Each required parallel class is also listed in a compressed manner. Any automorphism of order 3 on the Kirkman system must either fix a parallel class, or must move the entire parallel class. If it fixes the parallel class, it may either fix the blocks of the parallel class, or permute them within the parallel class. In general, for each parallel class, some subgroup of the group of order 27 acting on the design fixes that parallel class. Hence it suffices to prescribe orbit representatives of the blocks in the parallel class in its stabilizing subgroup.

Each parallel class can therefore be succinctly described using the generators from $\S 2$ to specify the stabilizer of the parallel class, and listing orbit representatives for the parallel class under this subgroup. We specify the stabilizer of the parallel class by specifying a subgroup code, which is an integer from $\{0, \ldots, 7\}$. It is interpreted as follows. For the two groups having all elements of order 3 , let $\pi_{1}, \pi_{2}, \pi_{3}$ be the three generators (in the same order) as given in $\S 2$. For the two groups requiring two generators, let $\pi_{2}, \pi_{3}$ be the two generators as given in $\S 2$, and let $\pi_{1}=\pi_{2}^{3}$. For $Z_{27}$, let $\pi_{3}$ be the generator given in $\S 2, \pi_{2}=\pi_{3}^{3}$ and $\pi_{1}=\pi_{3}^{9}$. It is important to remark that an automorphism of order 9 may move a parallel class, while the cube of the same automorphism fixes it; hence, we require these redundant generators in specifying the stabilizer of each parallel class.

Now with permutations chosen in this way, write the subgroup code as a 3-bit binary number $b_{3} b_{2} b_{1}$; the stabilizer is found by generating the minimal subgroup containing $\pi_{1}^{b_{1}}, \pi_{2}^{b_{2}}$, and $\pi_{3}^{b_{3}}$. Each parallel class is written as a subgroup code, followed by orbit representatives for the parallel classes; parallel classes are separated in the listing by colons.

Therefore, to recover the entire resolution, one first finds the stabilizer for each parallel class in turn, and applies all permutations in the stabilizer to reconstruct the parallel class. Then one applies the action of the entire group to find all thirteen parallel classes.

We give an example of the process here. Over the second nonabelian group, the following is a compact representation of a Kirkman triple system: 4 adv e $j$ $f m z: 6$ anz: $2 f n u$. There are three orbits of parallel classes. To recover the orbit representatives for all parallel classes, apply the second generator to $a d v, e g j, f m z$, both generators to $a n z$, and the first generator to $f n u$. This yields the three parallel classes:

$$
\begin{aligned}
& \text { adv bxs chr egj tuA lop fmz kqw iny } \\
& \text { anz dot epA bfq gru hsv ciw jlx kmy } \\
& \text { fnu gov hpw iqx jry ksz act dlA bem }
\end{aligned}
$$

Now apply both generators to produce all parallel classes in the orbits of these three. The orbit lengths obtained are 9,1 , and 3 for the three parallel classes given: 


$$
\begin{aligned}
& \text { adv bxs chr egj tuA lop fmzkqw iny } \\
& \text { dewcuy ils fhk bvA mpq gnt arx joz } \\
& \text { efx lvz cjm agi buw nqr hoA dsy kpt } \\
& \text { fgy mtw kln dhj uvx ors bip cez aqA } \\
& \text { ghz nxA amo eik vwy cps jqu flt bdr } \\
& \text { hit boy dnp afj wxz clq krv gmA esu } \\
& \text { ijA puz eoq dgk txy lmr asw bhn cfv } \\
& \text { bjk qtv fpr aeh yzA mns cdx iou glw } \\
& \text { akurwA gqs dfi btz cno ely jpv hmx } \\
& \text { anz dot epA bfq gru hsv ciw jlx kmy } \\
& \text { fnu gov hpw iqx jry ksz act dlA bem } \\
& \text { fow jst env irz dmu hqy abl gpx ckA } \\
& \text { jnw hlu fsA dqz kox imv bcg ert apy }
\end{aligned}
$$

\section{ACKNOWLEDGMENTS}

Research of the first author is supported by NSERC Canada under grant number A0579. Research of the second author is supported under NSA grant 88F066, and by the Center for Communications and Information Science, UNL. Research of the third author is supported by NSERC Canada under grant A8651.

\section{BIBLIOGRAPHY}

1. S. Bays, Sur les systèmes cycliques de triples de Steiner différent pour $n$ premier (ou puissance de nombre premier) de la forme $6 n+1$, Comment. Math. Helv. 4 (1932), 183-194.

2. M. J. Colbourn and R. A. Mathon, On cyclic Steiner 2-designs, Ann. Discrete Math. 7 (1980), 215-253.

3. M. Hall, Jr., The theory of groups, Macmillan, New York, 1959.

4. Z. Janko and T. van Trung, On projective planes of order 12 with an automorphism of order 13. Part 1: Kirkman designs of order 27, Geom. Dedicata 11 (1981), 257-284.

5. R. A. Mathon, K. T. Phelps, and A. Rosa, A class of Steiner triple systems of order 21 and associated Kirkman systems, Math. Comp. 37 (1981), 209-222.

6. B. D. McKay, Practical graph isomorphism (Proc. Tenth Manitoba Conf. Numerical Math. Computing), Congr. Numer. 30 (1981), 45-87.

7. K. Phelps and A. Rosa, Steiner triple systems with rotational automorphisms, Discrete Math. 33 (1981), 57-66.

8. V. D. Tonchev, Transitive Steiner triple systems of order 25, Discrete Math. 67 (1987), 211-214.

Department OF Combinatorics and Optimization, University of Waterloo, Waterloo, ONTARIO N2L 3G1, CaNADA

Department of Computer Science and ENGINeERING, University of Nebraska-Lincoln, LINCOLN, NEBRASKA 68858

Department of Computer SCIENCE, University of Toronto, Toronto, Ontario M5S 1A4, CANADA 


\title{
Supplement to
}

\section{TRANSITIVE STEINER AND KIRKMAN TRIPLE SYSTEMS OF ORDER 27}

\author{
CHARLES J. COLBOURN, SPYROS S. MAGLIVERAS, AND RUDOLF A. MATHON
}

\section{Transitive Kirkman Triple Systems of Order 27}

See $\S 3$ of that paper for instructions in reading the following tables.

\section{The nonabelian group of exponent three}

This group carries 146 nonisomorphic transitive Kirkman triple systems. We list them next, giving the number of the underlying transitive $S T S$ in the lists of $\S 2$, and the order of the automorphism group of the Kirkman triple system.

\begin{tabular}{|c|c|c|c|}
\hline No. & STS & Order & Representation \\
\hline 1 & 1 & 303264 & 6 alq bux chi: 5 amo dhr euv: 4 cjr dit asz: 4 clu etz aix:7 cno dlp \\
\hline 2 & 1 & 11664 & $\begin{array}{l}7 \text { ajp:7 amo:7 ant:7 cjr dmv:7 clu:7 cno dlp:7 cps:7 cy A dgj:7 fjl:7 fkz:7 } \\
\text { fuA:7 juy:7 los }\end{array}$ \\
\hline 3 & 1 & 3888 & 6 afg brs chi:5 amo dhr euv: 4 cjr dit asz: 4 clu etz aix:7 fjl \\
\hline 4 & 1 & 3888 & $\begin{array}{l}7 \text { ajp:5 amo dwA eis:4 arv bhq cdk:7 cjr dmv:7 cno dlp:7 cy A dgj:7 fjl:7 } \\
\text { fkz:7 fuA }\end{array}$ \\
\hline 5 & 2 & 3888 & 7 ajp:5 amo dhr euv:4 arv bhq cdk:6 cjw:7 cno dlp:7 cyA dgj:7 fjl \\
\hline 6 & 1 & 2916 & 7 ajp:5 amo dhr euv:4 cjr dit asz:4 clu etz aix:7 cno dlp:7 cy A dgj:7 fjl \\
\hline 7 & 1 & 2916 & 7 ajp:5 amo dhr euv:7 cjr dmv:7 clu:7 cno dlp:7 cps:7 cy A dgj:7 fjl:7 \\
\hline 8 & 1 & 2916 & $\begin{array}{l}7 \text { ajp:5 amo dwA eis: } 4 \text { arv bhq cdk:7 cno dlp:4 cqx dit akA:7 cyA dgj:7 } \\
\text { fjl }\end{array}$ \\
\hline 9 & 1 & 1944 & $\begin{array}{l}7 \text { ajp:5 amo dhr euv:4 arv bhq cdk:4 cjr dit asz:7 cno dlp:7 cy A dgj:7 } \\
\text { fjl }\end{array}$ \\
\hline 10 & 1 & 1944 & $\begin{array}{l}7 \text { ajp:5 amo dhr euv:4 cjr dit asz:7 clu:7 cno dlp:7 cps:7 cy A dgj:7 fjl:7 } \\
\text { los }\end{array}$ \\
\hline 11 & 2 & 1944 & $\begin{array}{l}7 \text { ajp:5 amo dhr euv:4 as A bip cex:7 clu:7 cno dlp:7 cps:7 cyA dgj:7 } \\
\text { fjl:7 los }\end{array}$ \\
\hline 12 & 1 & 972 & 6 afg brs chi:5 amo dwA eis:4 arv bhq cdk:4 cqx dit akA:7 fjl \\
\hline 13 & 1 & 972 & $\begin{array}{l}6 \text { afg brs chi:7 amo:7 ant:7 cjr dmv:7 clu:7 cps:7 fjl:7 fkz:7 fuA:7 juy:7 } \\
\text { los }\end{array}$ \\
\hline 14 & 1 & 972 & 6 afg bux chi: 5 amo dhr euv: 4 cjr dit asz: 4 clu etz aix:7 cy $A$ dgj \\
\hline 15 & 1 & 972 & 6 afg bux chi:5 amo dwA eis:4 arv bhq cdk:4 cqx dit akA:7 cyA dgj \\
\hline 16 & 1 & 972 & $\begin{array}{l}6 \text { afg bux chi:7 amo:7 ant:7 cjr dmv:7 clu:7 cps:7 cyA dgj:7 fkz:7 fuA:7 } \\
\text { juy:7 los }\end{array}$ \\
\hline 17 & 1 & 972 & $\begin{array}{l}7 \text { ajp:5 amo dhr euv:4 arv bhq cdk:7 cjr dmv:7 cno dlp:7 cy A dgj:7 fjl:7 } \\
\text { fkz:7 fuA }\end{array}$ \\
\hline 18 & 2 & 972 & 7 ajp:5 amo dhr euv:5 cjw dty:4 clu etz aix: 7 cno dlp:7 cy A dgj:7 fjl \\
\hline 19 & 2 & 972 & 7 ajp:5 amo dwA eis:4 arv bhq cdk:5 cjw dty:7 cno dlp:7 cyA dgj:7 fjl \\
\hline 20 & 2 & 486 & $\begin{array}{l}7 \text { ajp:5 amo dhr euv:4 arv bhq cdk:4 as A bip cex:7 cno dlp:7 cyA dgj:7 } \\
\text { fjl }\end{array}$ \\
\hline 21 & 2 & 486 & $\begin{array}{l}7 \text { ajp:5 amo dhr euv:4 as A bip cex:4 clu etz aix:7 cno dlp:7 cy A dgj:7 } \\
\text { fjl }\end{array}$ \\
\hline 22 & 4 & 432 & 7 akA:5 amo dlp euv:5 ckw dqt:5 clq:7 cps:7 fjl:7 juy \\
\hline
\end{tabular}




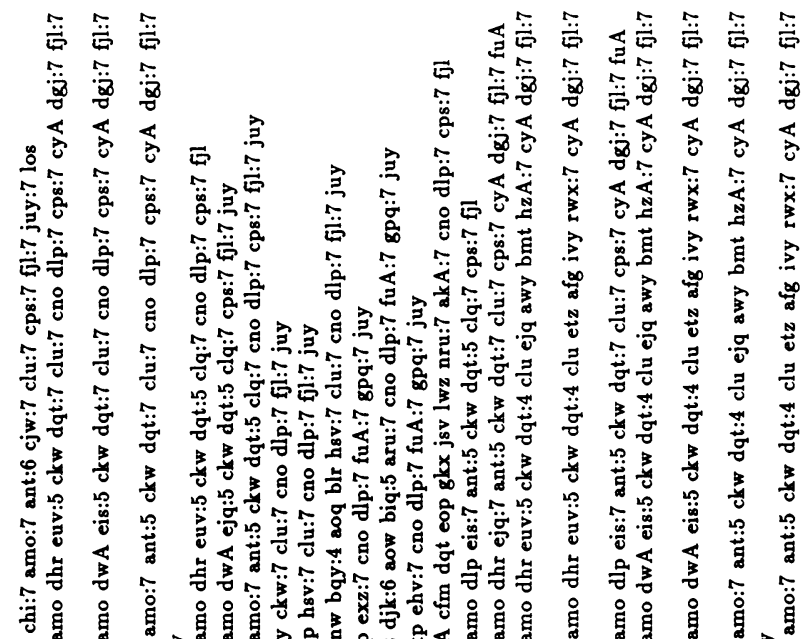

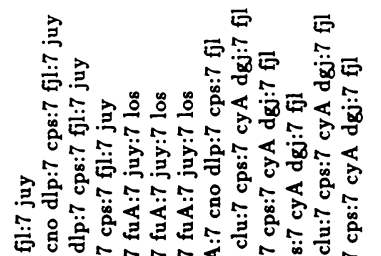

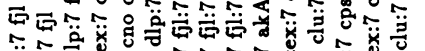

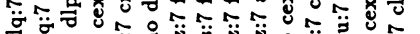

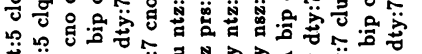

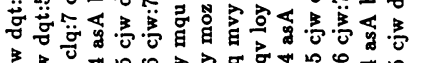

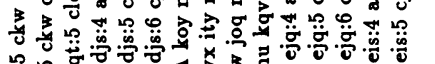

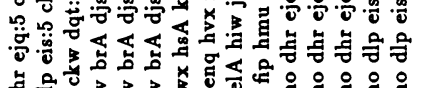

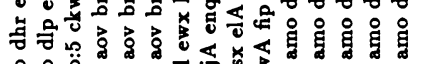

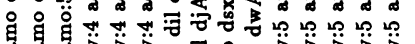
等

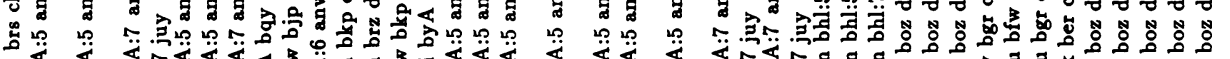

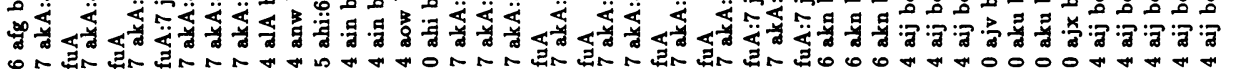

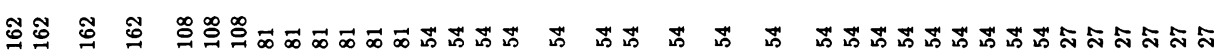

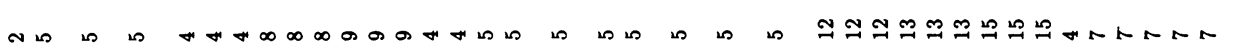

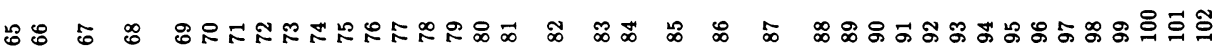

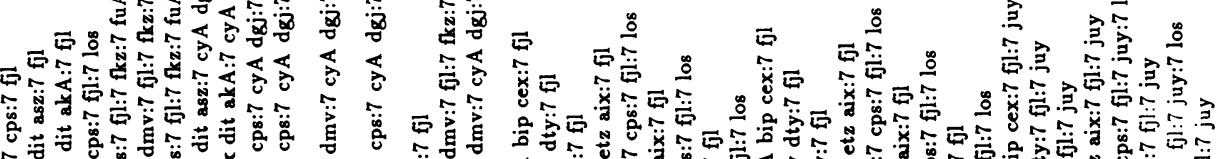

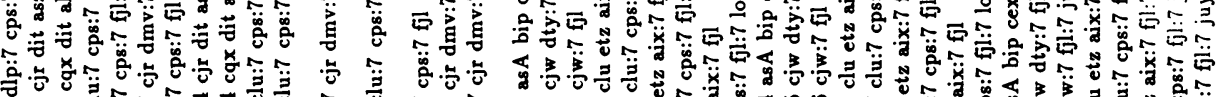

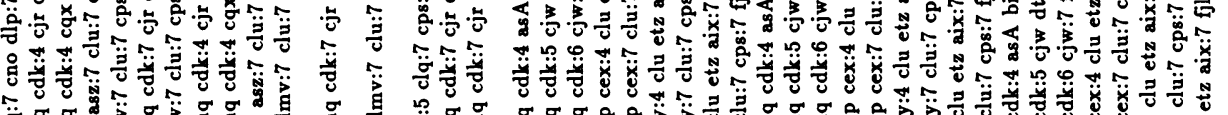

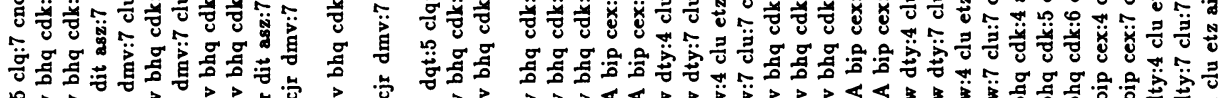

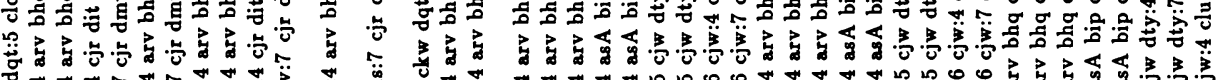

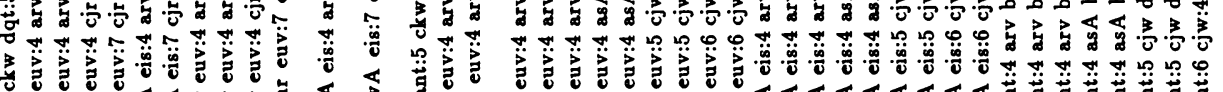

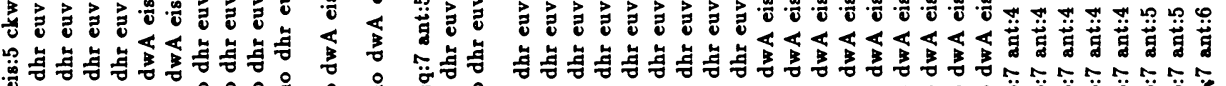

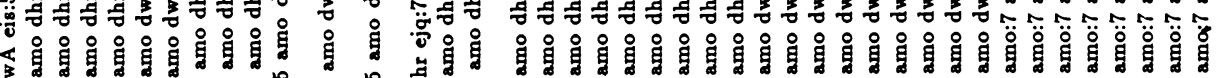
舟象

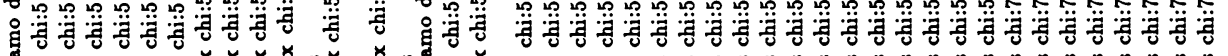

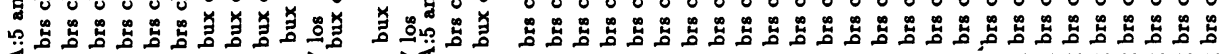

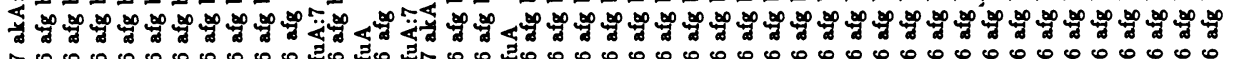

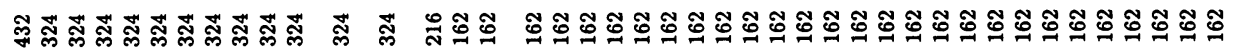



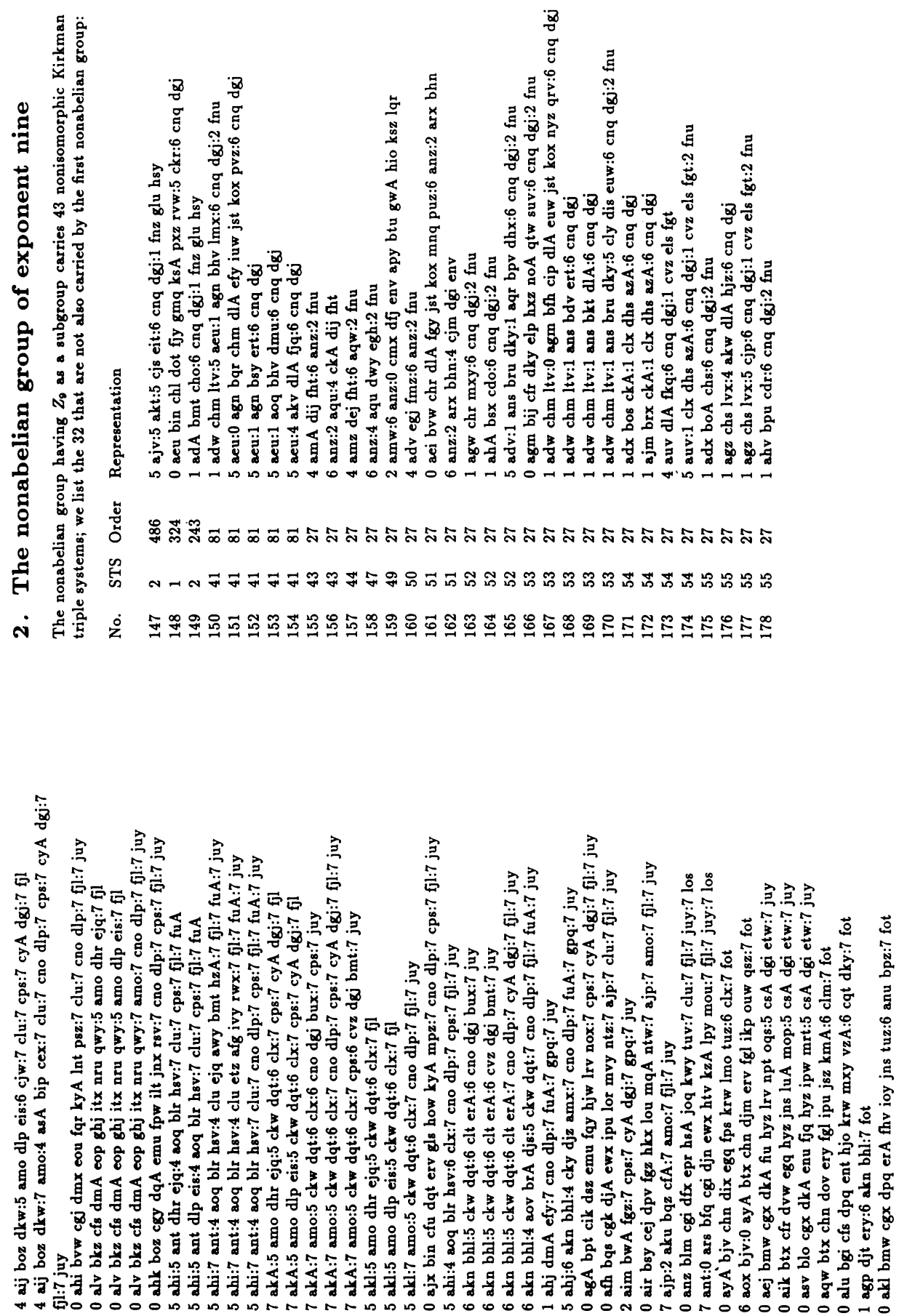

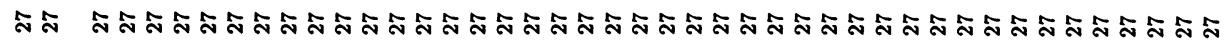

ヘ

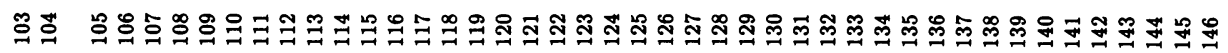



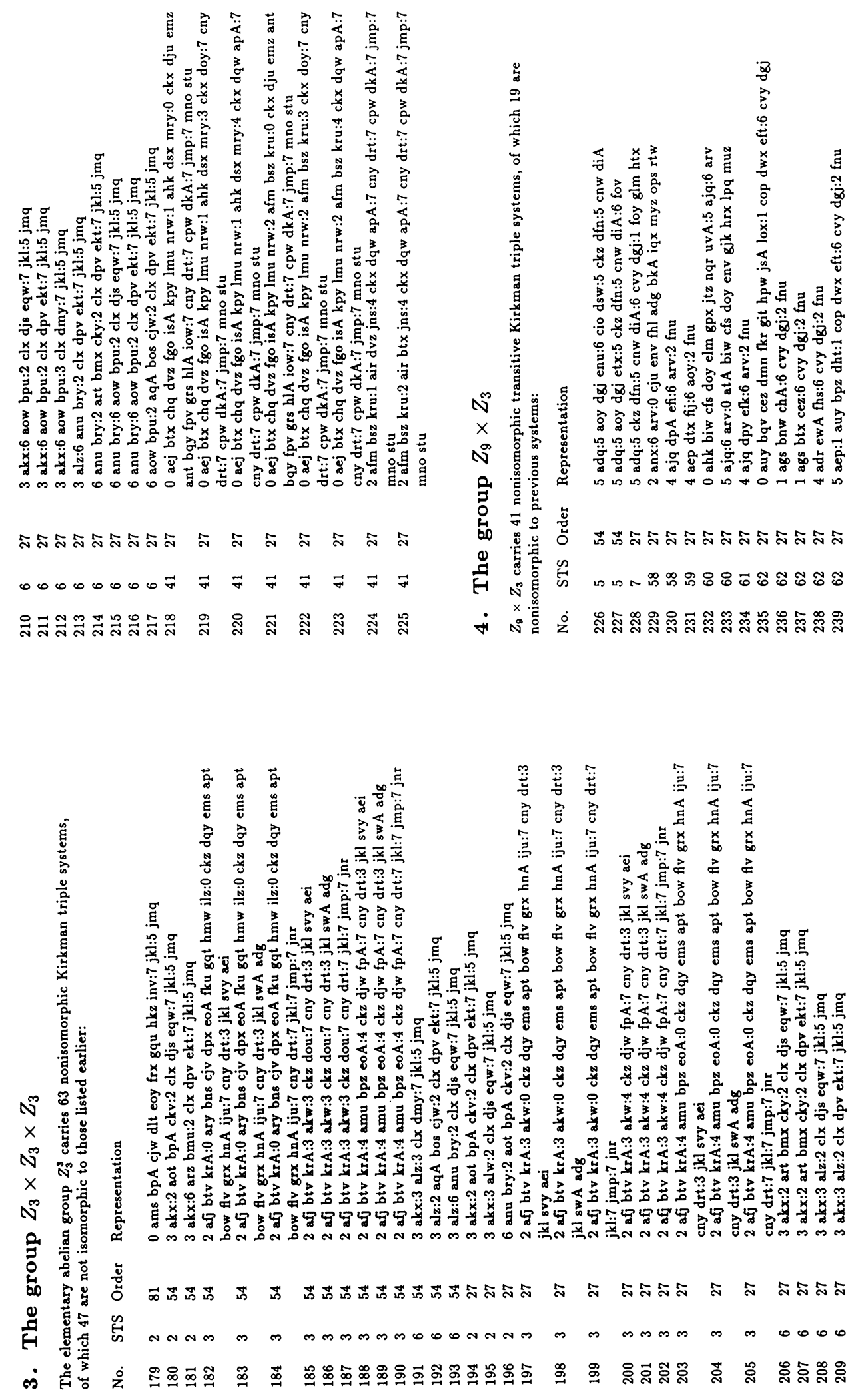


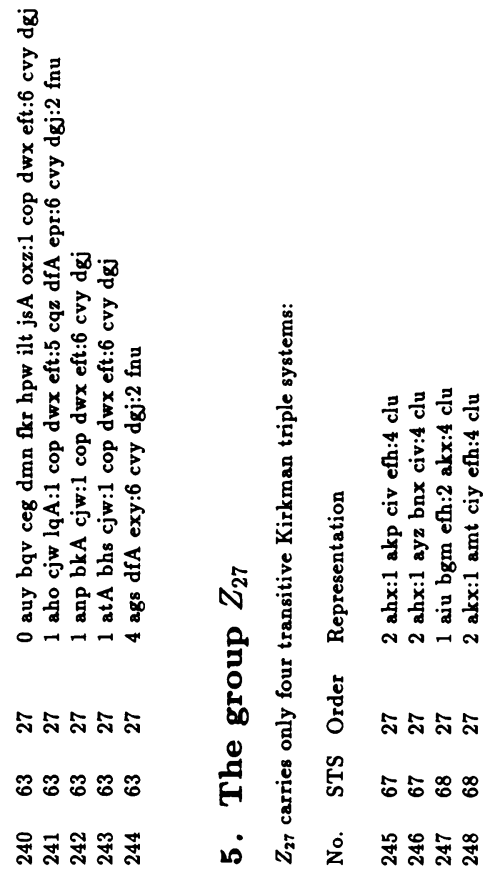

